\title{
Amplification by Frequency Transposition with a Low Critical Temperature DC SQUID
}

\author{
O. Hubert, Y. Monfort, J. Lepaisant, C. Gunther and D. Bloyet \\ Groupe de Recherche en Informatique, Image et Instrumentation de Caen (G.R.E.Y.C.)( ${ }^{*}$ ), \\ ISMRA, 6 Boulevard du maréchal Juin, 14050 Caen Cedex, France
}

(Received 3 November 1994, accepted 5 October 1995)

PACS.07.55+x - Magnetic instruments and techniques

\begin{abstract}
The performances of a heterodyne amplifier involving a DC SQUID have been modelised and measured. This type of amplification is derived from frequency translation methods using flux modulation. The pump frequency used depends on the signal to be analysed and the translation to a fixed frequency $f_{0}$ is carried out by the SQUID which is a nonlinear device. Such a system simplifies the impedance matching between the SQUID and the semiconductor preamplifier and can be operated as a spectrum analyser for signals of relatively high and variable frequency. Flux sensitivities of 5 to $10 \mu \Phi_{0} / \sqrt{\mathrm{Hz}}$ have been measured in this configuration for frequencies ranging from $200 \mathrm{kHz}$ to $2 \mathrm{MHz}$. In order to validate our model, the flux sensitivity of the SQUID in the heterodyne configuration has been compared to that given by direct measurements at the intermediate frequency $f_{0}$. Our measurements are in good agreement with a theoretical model based on a simplified voltage to flux characteristic of the DC SQUID.
\end{abstract}

\section{Introduction}

Many low noise preamplifying systems associating a DC SQUID (Direct Current Superconducting Quantum Interference Device) and a semiconductor preamplifier have been proposed. In these systems, an impedance matching of the SQUID output impedance ( 1 to $10 \Omega$ ) to the optimal input impedance of the semiconductor preamplifier (several $k \Omega$ or tens of $k \Omega$ ) is needed. For low frequency applications, one can use the nonlinearity of the SQUID response to flux $V(\Phi)$ to translate the signal spectrum at a higher frequency by the application of an auxiliary magnetic flux [1]. The coupling of the SQUID to the preamplifier can be then selective and furthermore, the low frequency noise of $1 / f$ can be eliminated. Most of the classical flux modulation devices are associated to a flux control loop, called Flux Locked Loop (FLL), and use a series resonant circuit [1] or a cooled transformer [2-5] for the impedance matching. Preferring to optimize the magnetic field sensitivity rather than the energy resolution, Cantor et al. [6] managed to achieve a direct coupling between the SQUID and the preamplifier. A more sophisticated system includes a cooled semiconductor preamplifier [7] which amplifies the signal at low temperature and reduces the size of the measurement system. Another original device achieves the impedance transformation with two SQUIDS in cascade [8] and a positive feedback system, proposed by Seppä et al. [9], makes the amplifier noise negligible but can become unstable for frequencies higher than $150 \mathrm{kHz}$. Furthermore, many authors [10-12] have

$\left(^{*}\right)$ URA CNRS 1526.

(C) Les Éditions de Physique 1996 
developed $1 / f$ noise reduction techniques involving flux modulation and/or SQUID bias current modulation. These realizations work in a relatively narrow band or at low frequencies and are well suited for NMR or NQR applications [13-15] and for gravitational wave detection [16]. Nevertheless, broadband preamplifiers (eventually coupled to an input resonant circuit of low damping factor) are also required for some other nuclear resonance measurement systems which operate on a wide range of high frequencies $[17,18]$. Moreover, the carrier frequencies have to be much higher than the signal frequency [19]. Thus, the semiconductor preamplifier noise dominates that of the SQUID in the frequency range $200 \mathrm{kHz}-10 \mathrm{MHz}$ in which we are concerned.

As compared to a semiconductor preamplifier, the noise temperature of a DC SQUID is proportional to the frequency and can be as low as $0.1 \mathrm{~K}$ at $10 \mathrm{MHz}$ for a SQUID inductance close to $200 \mathrm{pH}[20]$.

We have developed a preamplification technique derived from flux modulation methods so as to decrease the contribution of the semiconductor preamplifier noise over a large frequency range. The SQUID is excited by the heterodyne signal whose frequency depends on the signal. The translation of the signal spectrum results from the SQUID nonlinearity. The matching between the SQUID and the preamplifier is simplified as the intermediate frequency $f_{0}$, the working frequency, is fixed by the designer.

This method is presented in Section 2. The flux-voltage transfer coefficient (Section 2.1) and the contribution of the different noise sources (Section 2.2) are evaluated from an approximate expression of the SQUID response $V(\Phi)$. The flux sensitivity of the SQUID to applied auxiliary flux modulation is then calculated. Section 3 is devoted to experimental results obtained either by direct measurement or with a heterodyne amplifier. The influence of the noise sources on the system performances are studied in Section 4 .

\section{Principle of the Method}

The flux - voltage characteristic $V(\Phi)$ of a DC SQUID is periodic, with a period $\Phi_{0}$ ( $\Phi_{0}$ : quantum of flux), and a shape dependent on the bias current $I_{\mathrm{p}}$. Usually, $I_{\mathrm{p}}$ is adjusted so that the SQUID response is close to a sine curve and can be expressed by:

$$
V=A\left(1-\cos \left[\frac{2 \pi \Phi_{\mathrm{a}}}{\Phi_{0}}\right]\right)
$$

where $A$ is the signal amplitude at the DC SQUID output and $\Phi_{\mathrm{a}}$ the total flux applied (Fig. 1).

The amplification system based on SQUID is shown Figure 2. We are expecting to study the magnetization spectral response of a sample in continuous NMR or NQR spectrometry with this system and our heterodyne amplification method (especially developed for this application field).

The magnetization of the sample placed in the pick-up coil $L_{\mathrm{p}}$ induces a current in the coil $L_{\imath}$ coupled to the SQUID and the flux thus applied to the SQUID is converted into a voltage. In flux modulation systems, the amplitude of the pump signal $\alpha \Phi_{0}(\alpha \approx 0.25)$ is much higher than the useful signal. The modulating signal of frequency $f_{2}$ and centered on $\Phi_{0} / 2$ is injected by the modulation coil $L_{\mathrm{m}}$. The DC SQUID therefore operates as a mixer whose intermediate frequencies are $f_{1} \pm f_{2}$.

Furthermore, classical devices with flux modulation use a fixed frequency $f_{2}$, the preamplifier (A1) has therefore to work in the frequencies range going from $f_{1}-f_{2}$ to $f_{1}+f_{2}$. The original feature of our method is that the frequency $f_{2}$ is adjusted so that the constraint $f_{1}-f_{2}=f_{0}$ 


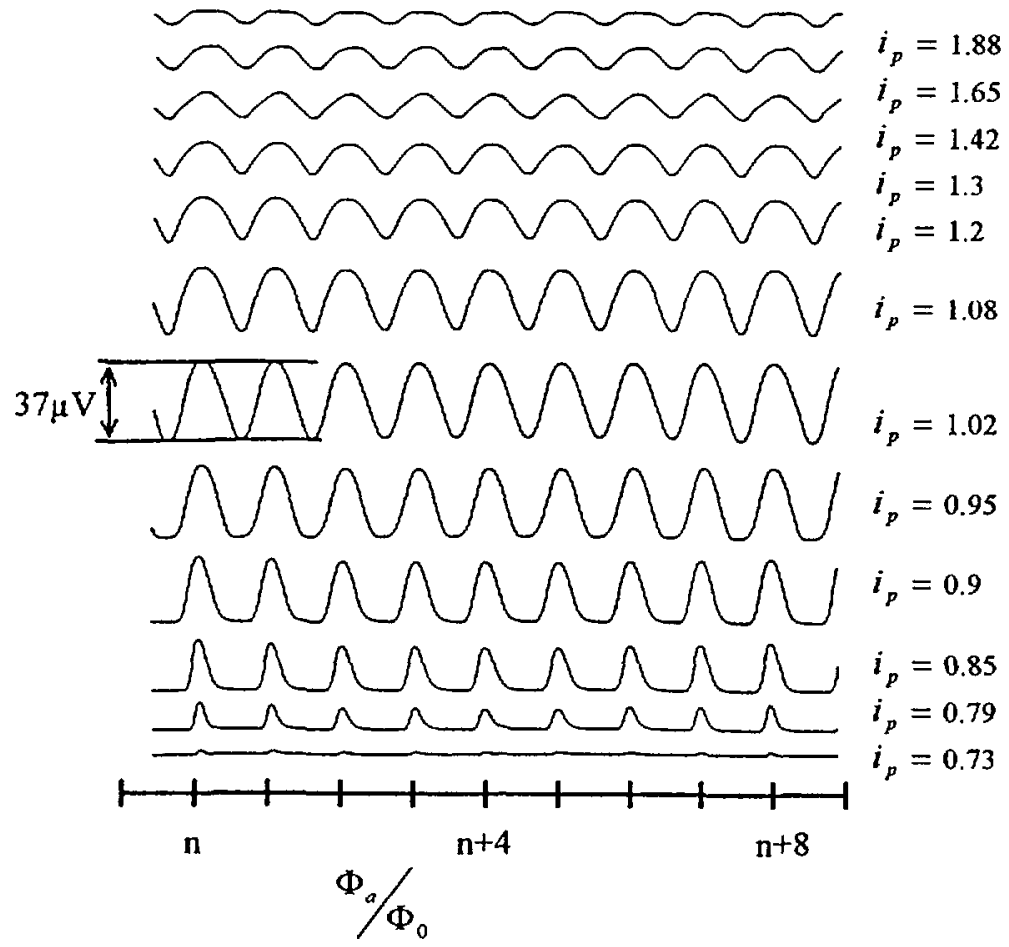

Fig. 1. - Voltage versus flux characteristics of the Conductus DC SQUID for different values of bias current $i_{\mathrm{p}}=\frac{I_{\mathrm{p}}}{2 I_{\mathrm{c}}}$, with $I_{\mathrm{c}}=12.5 \mu \mathrm{A}$.

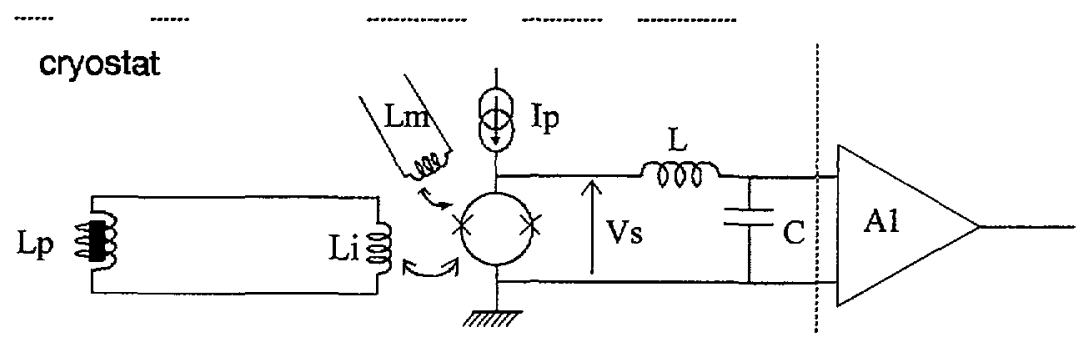

Fig. 2. - Schematics of the DC SQUID for amplifying the NMR or NQR signal of the pick up Loop $L_{\mathrm{p}}$.

is always satisfied. $f_{0}$ is the chosen central frequency and the signal component at $f_{0}$ is selected by the $L C$ resonant circuit cooled at low temperature. The signal is amplified at room temperature and lock-in detected to get back the SQUID signal.

2.1. Flux - Voltage Transfer Factor of the Ideal System. - Assuming a noiseless system, the voltage across the SQUID output results from the sum of the applied fluxes:

$$
V_{\mathrm{s}}=A\left(1-\cos \left[\frac{2 \pi}{\Phi_{0}}\left(\Phi_{\mathrm{B}}+\alpha \Phi_{0} \cos \left(\omega_{2} t\right)+\Delta \Phi \cos \left(\omega_{1} t+\delta\right)\right)\right]\right)
$$


where $\Phi_{\mathrm{B}}$ is the bias flux, $\Delta \Phi$ the amplitude of the signal which is assumed to be sinusoidal. $\Delta \Phi$ is very small compared to $\Phi_{0}$, i.e. $\frac{2 \pi \Delta \Phi}{\Phi_{0}} \ll 1$, so the expression (2) can be developed as Bessel's functions:

$$
V_{\mathrm{s}}=A-A\left[\begin{array}{l}
\cos \left(\frac{2 \pi \Phi_{\mathrm{B}}}{\Phi_{0}}\right)\left(J_{0}(2 \pi \alpha)-2 J_{2}(2 \pi \alpha) \cos \left(2 \omega_{2} t\right)+. .\right) \\
-\cos \left(\frac{2 \pi \Phi_{\mathrm{B}}}{\Phi_{0}}\right)\left(2 J_{1}(2 \pi \alpha) \cos \left(\omega_{2} t\right)+. .\right) \frac{2 \pi \Delta \Phi}{\Phi_{0}} \cos \left(\omega_{1} t+\delta\right) \\
-\sin \left(\frac{2 \pi \Phi_{\mathrm{B}}}{\Phi_{0}}\right)\left(J_{0}(2 \pi \alpha)-2 J_{2}(2 \pi \alpha) \cos \left(2 \omega_{2} t\right)+. .\right) \frac{2 \pi \Delta \Phi}{\Phi_{0}} \cos \left(\omega_{1} t+\delta\right) \\
-\sin \left(\frac{2 \pi \Phi_{\mathrm{B}}}{\Phi_{0}}\right)\left(2 J_{1}(2 \pi \alpha) \cos \left(\omega_{2} t\right)+. .\right)
\end{array}\right]
$$

The $L C$ circuit connected with the preamplifier input selects the component of pulsation $\omega_{2}-\omega_{1}=\omega_{0}$ (second line of the expression (3)). The resulting voltage $V_{s}\left(\omega_{0}\right)$ at the SQUID output is expressed as:

$$
V_{\mathrm{s}}\left(\omega_{0}\right)=A \cos \left(\frac{2 \pi \Phi_{\mathrm{B}}}{\Phi_{0}}\right) J_{1}(2 \pi \alpha) \frac{2 \pi \Delta \Phi}{\Phi_{0}} \cos \left(\omega_{0} t-\delta\right)
$$

Under these conditions, $V_{\mathrm{s}}\left(\omega_{0}\right)$ is maximal for $\alpha \approx 0.29$ and $\Phi_{\mathrm{B}}=n \Phi_{0} / 2$ with $n$ integer. From the expression (1) relative to a direct measurement, the flux - voltage transfer coefficient is equal to $2 \pi A$ when $\Phi_{\mathrm{B}}=\left(\frac{1}{4}+\frac{n}{2}\right) \Phi_{0}$. The application of flux modulation decreases this value of a factor $J_{1}(2 \pi \alpha)=0.58$ for $\alpha=0.29$.

2.2. Effects of the Different Sources of Noise. - The main sources of noise in our experimental set-up are the SQUID intrinsic noise and the equivalent noise voltage $e_{\text {na }}(t)$ of the semiconductor amplifier referred to the SQUID output. As usual, the SQUID intrinsic noise is supposed to be only due to the Johnson noise in the resistive shunts, called $R$, of the junctions. The SQUID is affected in two ways: a voltage noise $v_{n}(t)$ is induced in series with the SQUID and a flux noise $\varphi_{\mathrm{n}}(t)$ is applied to the SQUID inductance $L_{\mathrm{s}}$. For bias current higher than the critical current, the voltage noise power spectra $[21,22]$ of these noise terms are respectively:

$$
\begin{aligned}
& \overline{v_{n}^{2}(\omega)} \approx 2 R_{\mathrm{d}}^{2}\left(\frac{4 k_{\mathrm{B}} T}{R}\right) \\
& \overline{\varphi_{\mathrm{n}}^{2}(\omega)} \approx L_{\mathrm{s}}^{2} \frac{4 k_{\mathrm{B}} T}{2 R}
\end{aligned}
$$

where $k_{\mathrm{B}}$ is the Boltzmann's constant, $L_{\mathrm{s}}$ the SQUID inductance and the $R_{\mathrm{d}}$ SQUID dynamic resistance defined by $R_{\mathrm{d}}=\left.\frac{\partial V_{\mathrm{s}}}{\partial I_{\mathrm{p}}}\right|_{\Phi_{\mathrm{a}}} ; R_{\mathrm{d}}$ can vary from $R$ to $3 R$ for usual bias conditions.

Furthermore, a phase noise $\theta_{\mathrm{n}}(t)$ can also be introduced by the synthesized generator which supplies the pump signal [23]. As opposed to the other noise sources, the phase noise is white since its amplitude is maximal around the pump frequency $f_{2}$ and decreases drastically above. $\theta_{\mathrm{n}}^{2}(\omega)$ is the power spectral density at the pulsation $\omega$ and is expressed in $\operatorname{rad}^{2} / \mathrm{Hz}$. Finally, the noise associated with the signal will be assumed to be negligible.

In the absence of a signal, the output voltage noise is

$$
V_{\mathrm{sb}}(t)=v_{\mathrm{n}}(t)+e_{\mathrm{na}}(t)+A\left(1-\cos \left[\frac{2 \pi}{\Phi_{0}}\left(\Phi_{\mathrm{B}}+\alpha \Phi_{0} \cos \left(\omega_{2} t+\theta_{\mathrm{n}}(t)\right)+\varphi_{\mathrm{n}}(t)\right)\right]\right)
$$


Assuming that $\frac{2 \pi \Phi_{n}(t)}{\Phi_{0}} \ll 1$ and $\theta_{n}(t) \ll 1$ and developing on the basis of Bessel's functions, we obtain:

$$
-A\left[\begin{array}{l}
V_{\mathrm{sb}}(t)=v_{\mathrm{n}}(t)+e_{\mathrm{na}}(t)+A \\
\cos \left(\frac{2 \pi \Phi_{\mathrm{B}}}{\Phi_{0}}\right)\left(J_{0}(2 \pi \alpha)-2 J_{2}(2 \pi \alpha) \cos \left(2 \omega_{2} t\right)+. .\right) \\
-\cos \left(\frac{2 \pi \Phi_{\mathrm{B}}}{\Phi_{0}}\right)\left(2 J_{1}(2 \pi \alpha) \cos \left(2 \omega_{2} t\right)+. .\right)\left(-\alpha \Phi_{0} \theta_{\mathrm{n}}(t) \sin \left(\omega_{2} t\right)+\varphi_{\mathrm{n}}(t)\right) \frac{2 \pi}{\Phi_{0}} \\
-\sin \left(\frac{2 \pi \Phi_{\mathrm{B}}}{\Phi_{0}}\right)\left(J_{0}(2 \pi \alpha)-2 J_{2}(2 \pi \alpha) \cos \left(2 \omega_{2} t\right)+. .\right)\left(-\alpha \Phi_{0} \theta_{\mathrm{n}}(t) \sin \left(\omega_{2} t\right)+\varphi_{\mathrm{n}}(t)\right) \frac{2 \pi}{\Phi_{0}} \\
-\sin \left(\frac{2 \pi \Phi_{\mathrm{B}}}{\Phi_{0}}\right)\left(2 J_{1}(2 \pi \alpha) \cos \left(\omega_{2} t\right)+. .\right)
\end{array}\right]
$$

For a bias flux $\Phi_{\mathrm{B}}$ equal to $\frac{n}{2} \Phi_{0}$, the SQUID output voltage (at the pulsation $\omega_{2}-\omega_{1}$ ) comes from the second line of the square bracket $(8)$ :

$$
\begin{aligned}
& V_{\mathrm{sb}}(t)=v_{\mathrm{n}}(t)+e_{\mathrm{na}}(t) \\
& \pm \frac{2 \pi A}{\Phi_{0}}\left\{2 J_{1}(2 \pi \alpha) \cos \left(\omega_{2} t\right)-2 J_{3}(2 \pi \alpha) \cos \left(3 \omega_{2} t\right) . .\right\}\left\{-\alpha \Phi_{0} \theta_{\mathrm{n}}(t) \sin \left(\omega_{2} t\right)+\varphi_{\mathrm{n}}(t)\right\}
\end{aligned}
$$

From this noise model, the average quadratic value of $V_{\mathrm{sb}}(t)$ at the pulsation $\omega_{0}=\omega_{2}-\omega_{1}$ can be evaluated as:

$$
\begin{aligned}
& \overline{V_{\mathrm{sb}}^{2}\left(\omega_{0}\right)}= \overline{v_{\mathrm{n}}^{2}\left(\omega_{0}\right)}+\overline{e_{\mathrm{na}}^{2}\left(\omega_{0}\right)}+\left(\frac{2 \pi A}{\Phi_{0}}\right)^{2}\left[\begin{array}{l}
J_{1}^{2}(2 \pi \alpha)\left\{\overline{\varphi_{\mathrm{n}}^{2}\left(\omega_{2}-\omega_{0}\right)}+\overline{\varphi_{\mathrm{n}}^{2}\left(\omega_{2}+\omega_{0}\right)}\right\} \\
+J_{3}^{2}(2 \pi \alpha)\left\{\overline{\varphi_{\mathrm{n}}^{2}\left(3 \omega_{2}-\omega_{0}\right)}+\overline{\varphi_{\mathrm{n}}^{2}\left(3 \omega_{2}+\omega_{0}\right)}\right\} . .
\end{array}\right] \\
&+(\pi \alpha A)^{2}\left[\begin{array}{l}
\left\{J_{1}(2 \pi \alpha)+J_{3}(2 \pi \alpha)\right\}^{2}\left\{\overline{\theta_{\mathrm{n}}^{2}\left(2 \omega_{2}+\omega_{0}\right)}+\overline{\theta_{\mathrm{n}}^{2}\left(2 \omega_{2}-\omega_{0}\right)}\right\} \\
+\left\{J_{3}(2 \pi \alpha)+J_{5}(2 \pi \alpha)\right\}^{2}\left\{\overline{\theta_{\mathrm{n}}^{2}\left(4 \omega_{2}+\omega_{0}\right)}+\overline{\theta_{\mathrm{n}}^{2}\left(4 \omega_{2}-\omega_{0}\right)}\right\} . .
\end{array}\right]
\end{aligned}
$$

Compared to the $J_{3}$ and $J_{5}$ terms, the term $J_{1}(2 \pi \alpha)$ is predominant for $\alpha \approx 0.25$. Moreover, the pulsations $2 \omega_{2}+\omega_{0}$ and $2 \omega_{2}-\omega_{0}$ are close to the second harmonic of the pump pulsation $\omega_{2}$. The phase noise can therefore considered as white, i.e. $\overline{\theta_{\mathrm{n}}^{2}\left(2 \omega_{2}+\omega_{0}\right)} \approx \overline{\theta_{\mathrm{n}}^{2}\left(2 \omega_{2}-\omega_{0}\right)}$. From these approximations and expressions (4) and (10), the ratio (in $\sqrt{\mathrm{Hz}} / \Phi_{0}$ ) of the flux - 
voltage transfer coefficient to the effective voltage noise across the SQUID at the pulsation $\omega_{0}$ is deduced:

$$
\begin{aligned}
& \frac{\partial \overline{V_{\mathrm{s}}} / \partial \Phi_{\mathrm{a}, \mathrm{mod}}}{\sqrt{\overline{V_{\mathrm{sb}}^{2}\left(\omega_{0}\right)}}}= \\
& \frac{2 \pi A J_{1}(2 \pi \alpha)}{\sqrt{\overline{v_{\mathrm{n}}^{2}\left(\omega_{0}\right)}}+\overline{e_{\mathrm{na}}^{2}\left(\omega_{0}\right)}+2\left\{\pi A J_{1}(2 \pi \alpha)\right\}^{2}\left\{\left(\frac{2}{\Phi_{0}}\right)^{2} \overline{\varphi_{\mathrm{n}}^{2}\left(\omega_{2}-\omega_{0}\right)}+\overline{\alpha^{2} \theta_{\mathrm{n}}^{2}\left(2 \omega_{2}-\omega_{0}\right)}\right\}}
\end{aligned}
$$

The previous expression can be compared to the following expression obtained in the direct mode for $\Phi_{B}=\left(\frac{1}{4}+\frac{n}{2}\right) \Phi_{0}$ :

$$
\frac{\partial \overline{V_{\mathrm{s}}} / \partial \Phi_{\mathrm{a}}}{\sqrt{\overline{V_{\mathrm{sb}}^{2}\left(\omega_{1}\right)}}}=\frac{2 \pi A}{\sqrt{\overline{v_{\mathrm{n}}^{2}\left(\omega_{1}\right)}}+\overline{e_{\mathrm{na}}^{2}\left(\omega_{1}\right)}+\left(\frac{2 \pi A}{\Phi_{0}}\right)^{2} \overline{\varphi_{\mathrm{n}}^{2}\left(\omega_{1}\right)}}
$$

The correlation term between $v_{\mathrm{n}}(t)$ and $\varphi_{\mathrm{n}}(t)$ is zero in expressions (10) and (11): their respective contribution to the SQUID voltage noise comes from different pulsations $\omega_{0}$ and $\omega_{2} \pm \omega_{0}$ whatever the model employed to describe the SQUID behaviour. In the direct mode $\left(\Phi_{\mathrm{B}}=\left(\frac{1}{4}+\frac{n}{2}\right) \Phi_{0}\right.$ and $\left.f_{0}=f_{1}\right)$, our model is based on a symmetrical representation of the SQUID flux voltage characteristic so, it is not affected by the correlation term (12). Tesche and Clarke [24] have shown that this correlation coefficient is weak.

When an appropriate resonant circuit is associated with a low noise amplifier, $\overline{e_{\mathrm{na}}^{2}\left(\omega_{0}\right)}$ is very small compared to the other noise sources (11) (see Sect. 3). Furthermore, a low noise synthesized generator can make $\overline{\theta^{2}(\omega)}$ negligible (Sect. 4.3). In such a background, it results from the expressions (5), (6) and (11) that:

$$
\frac{\partial \overline{V_{\mathrm{s}}} / \partial \Phi_{\mathrm{a}, \mathrm{mod}}}{\sqrt{\overline{V_{\mathrm{sb}}^{2}\left(\omega_{0}\right)}}}=\frac{2 \pi A J_{1}(2 \pi \alpha)}{\sqrt{8 k_{\mathrm{B}} T \frac{R_{\mathrm{d}}^{2}}{R}+\left\{\frac{4 \pi A J_{1}(2 \pi \alpha)}{\Phi_{0}}\right\}^{2} L_{\mathrm{s}}^{2} \frac{k_{\mathrm{B}} T}{R}}}
$$

From equations (5), (6) and (12), the expression obtained for a direct measurement is:

$$
\frac{\partial \overline{V_{\mathrm{s}}} / \partial \Phi_{\mathrm{a}}}{\sqrt{\overline{V_{\mathrm{sb}}^{2}\left(\omega_{1}\right)}}}=\frac{2 \pi A}{\sqrt{8 k_{\mathrm{B}} T \frac{R_{\mathrm{d}}^{2}}{R}+\overline{e_{\mathrm{na}}^{2}\left(\omega_{1}\right)}+2\left\{\frac{2 \pi A}{\Phi_{0}}\right\}^{2} L_{\mathrm{s}}^{2} \frac{k_{\mathrm{B}} T}{R}}}
$$

It can be noticed that $\overline{e_{\text {na }}^{2}\left(\omega_{1}\right)}$ is not negligible when the application requires a wide band preamplifier. 


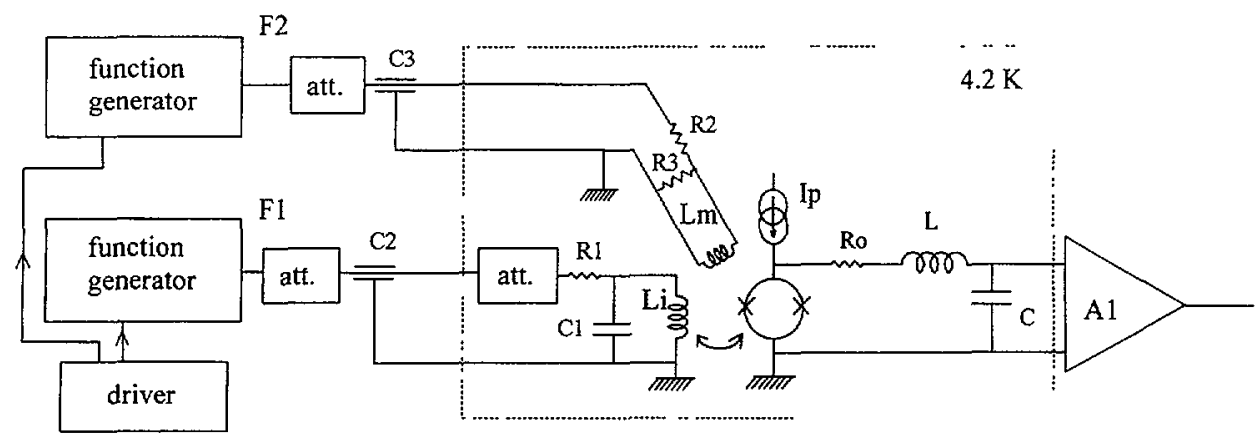

Fig. 3. - Experimental configuration which permits the simulation of the sample signal through $L_{\imath}$. It also allows to apply an external magnetic flux through $L_{\mathrm{m}}$ and to amplify the signal at chosen frequency; with $L=2.85 \mathrm{mH}$ and $C=7.25 \mathrm{nF}$ for $f_{0}=35 \mathrm{kHz}$.

\section{Experimental Device and Measurements}

3.1. Experimental Device. - The experimental device is represented in Figure 3. The SQUID voltage is measured by means of a resonant circuit cooled at $4.2 \mathrm{~K}$. The tank frequency, $f_{0}=35 \mathrm{kHz}$, is such that $f_{0}=f_{2}-f_{1} \ll f_{1}, f_{2}$ and the measurement chain operates beyond the $1 / f$ region. For a SQUID bias current $I_{\mathrm{p}}$ largely inferior to the critical current, the $Q$ factor is limited to a maximum value $Q_{\max } \approx 150$ by inserting a resistance $R_{0}=4.5 \Omega$ in series with the superconducting coil (niobium wires). This resistance is high enough to ensure the stability of the amplification system and its voltage noise $(32 \mathrm{pV} / \sqrt{\mathrm{Hz}})$ slightly affects the SQUID performance. $A 1$ is a low noise amplifier (JFET) developed at the laboratory, having the following noise characteristics: $e_{\mathrm{n}}=2.3 \mathrm{nV} / \sqrt{\mathrm{Hz}}$ and $i_{\mathrm{n}} \approx 10 \mathrm{f} \mathrm{A} / \sqrt{\mathrm{Hz}}$ at $35 \mathrm{kHz}[25]$.

The usual quality factor is close to 80 so that the voltage noise across the SQUID $(28 \mathrm{pV} / \sqrt{\mathrm{Hz}})$ is negligible. The SQUID is protected from ambient radiations at high frequencies by the resistances $R_{1}, R_{2}, R_{3}$ and capacitors $C_{1}, C_{2}, C_{3}$. Furthermore, the coupling coil $L_{2}$ has to be appropriately decoupled; this precaution appreciably reduces the resonance phenomena due to the parasitic capacitor between the coupling coil and the SQUID [3,26-29]. The SQUID used is a commercial DC SQUID (Conductus SQD1075), a Jaycox and Ketchen's type [30]. The relevant characteristics measured at $T=4.2 \mathrm{~K}$ are: and $R=5 \Omega, L_{\mathrm{s}}=170$ $\mathrm{pH}$ and $I_{\mathrm{c}}=12.5 \mu \mathrm{A}$ where $I_{\mathrm{c}}$ is the critical current of one junction. The performances of the SQUID amplifier have been studied when a sinusoidal pump signal at frequencies ranging from few hundred $\mathrm{kHz}$ to a few $\mathrm{MHz}$ is applied. More detailed measurements have been carried out at $f_{2}=535 \mathrm{kHz}$. The pump signal amplitude and the bias flux (in the vicinity of $0.5 \Phi_{0}$ ) have been optimized at each time. The signal to be analysed has been simulated by a small sinusoidal signal of amplitude $\Phi_{0} / 400$ at $f_{1}=500 \mathrm{kHz}$ (Fig. 5). These results have been compared to those obtained without auxiliary flux modulation for a bias flux of $0.25 \Phi_{0}$ or $0.75 \Phi_{0}$ (Fig. 4).

The SQUID performances calculated with our theoretical model (Sect. 2) are represented in Figures 4 and 5 . These semi-theoretical performances are calculated from dynamic resistances and amplitudes $A$ of the experimentally measured output signal.

3.2. Direct Measurement and Validity Domain of our Model. - Figure 4 shows the weak asymmetry of the response curve of the used SQUID since the coefficient $V_{\Phi}$ is higher for $\Phi_{\mathrm{a}}=0.75 \Phi_{0}$ than for $\Phi_{\mathrm{a}}=0.25 \Phi_{0}$. This particularity is clearly observed for a reduced current 


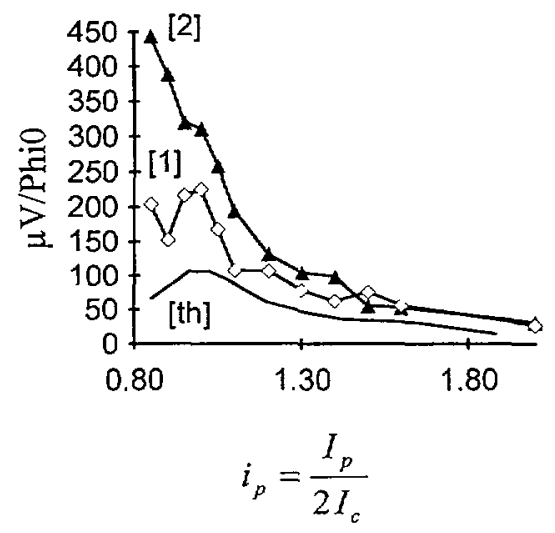

a)

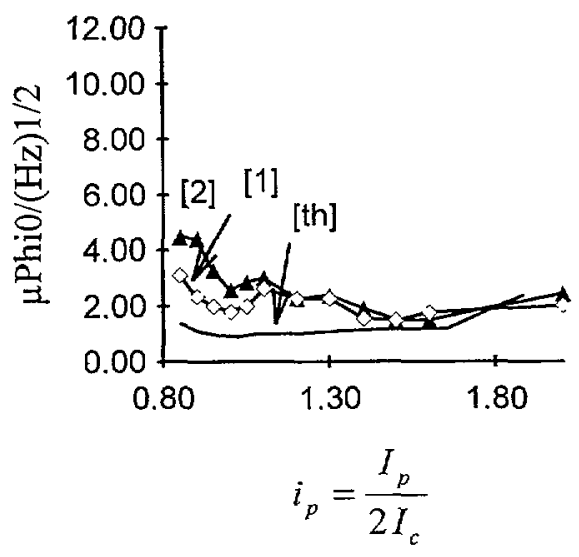

Fig. 4. - Direct measurement at $35 \mathrm{kHz}$ for an applied flux of $0.25 \Phi_{0}$ [1] and $0.75 \Phi_{0}$ [2], plotted with semi-theoretical [th] curves: a) voltage-flux transfer function, b) output noise voltage of the SQUID and c) SQUID flux sensitivity.

${ }_{\mathrm{p}}=\frac{I_{\mathrm{p}}}{2 I_{\mathrm{c}}}<1$ where the SQUID operates in a very unstable zone, close to the hysteresis. In that case, the coefficient $V_{\Phi}$ reaches high values $\left(450 \mu \mathrm{V} / \Phi_{0}\right.$ for $\left.\Phi_{\mathrm{B}}=0.75 \Phi_{0}\right)$ and the voltage - flux response no longer corresponds to our theoretical model. However, the SQUID output noise is very important in this voltage zone (Figs. $4 \mathrm{~b}, 5 \mathrm{~b}$ ) and the SQUID flux sensitivity is deteriorated. For bias currents $i_{\mathrm{p}}>1.6$, the SQUID performances are very irregular as several crossings are observed on the $I-V$ characteristic. The working field of the amplifier is therefore in the range $1 \leq i_{\mathrm{p}} \leq 1.6$ where the SQUID flux sensitivity is optimal and the coefficient $V_{\Phi}$ high enough.

The best flux sensitivity of the SQUID measured in the direct mode, $1.5 \mu \Phi_{0} \sqrt{\mathrm{Hz}}$ at $i_{\mathrm{p}}=1.5$ (Fig. 4c), corresponds to an energy resolution $\varepsilon=70 \mathrm{~h}$. This flux sensitivity is slightly greater than the value calculated from expression (13). Indeed, the coefficient $V_{\Phi}=\frac{\partial \bar{V}_{\mathrm{s}}}{\partial \Phi_{\mathrm{a}}}$ and the SQUID noise voltage are underestimated in our theoretical model. Moreover, the circuit injecting the signal in the coupling coil generates some noise that limits the experimental flux sensitivity (Fig. 3). 

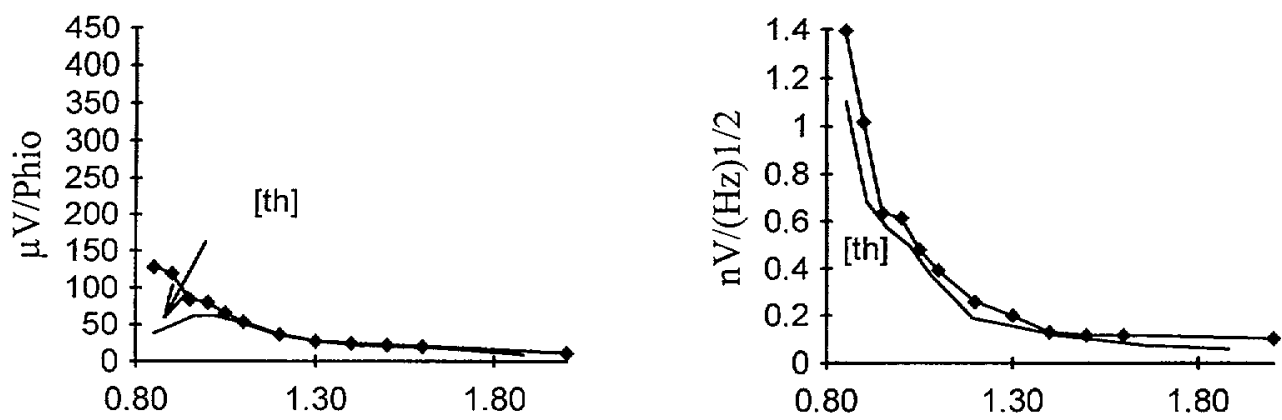

a)

$$
i_{p}=\frac{I_{p}}{2 I_{c}}
$$

b)

$$
i_{p}=\frac{I_{p}}{2 I_{c}}
$$

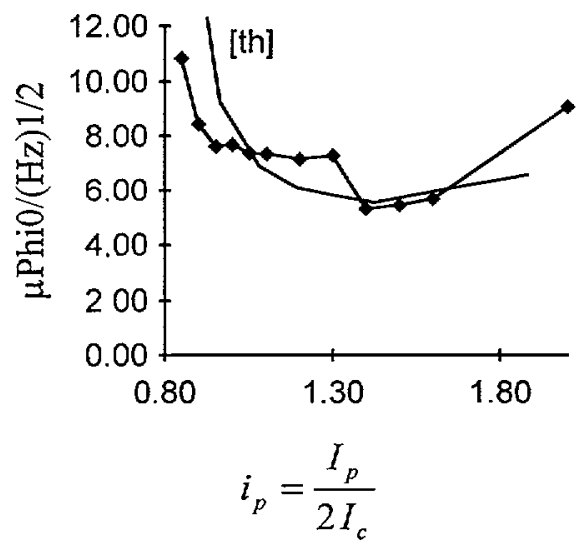

Fig. 5. - Measurement with pump signal at $535 \mathrm{kHz}$ for an applied flux of $0.5 \Phi_{0}$; semi-theoretical [th] and experimental curves: a) voltage-flux transfer function, b) output noise voltage of the SQUID and c) SQUID flux sensitivity.

The SQUID voltage noise (Fig, 4b) is higher than the theoretical expected value (14). These expressions only consider the noise in the resistances of the junctions. The noise increase due to mixing at the Josephson frequency $f_{\mathrm{J}}=\frac{V_{\mathrm{s}}}{\Phi_{0}}$ and its harmonics does not appear in our model [31]. This contribution decreases when the voltage $V_{\mathrm{s}}$ across the SQUID increases since the frequency goes away from the working frequency. Thereby, we experimentally observe a reduction of the SQUID noise voltage as the bias current increases.

Furthermore, the model developed in Section 2 does not take into account the strong coupling between the SQUID loop $L_{\mathrm{s}}$ and the coil $L_{\imath}$ (coupling coefficient around 0.78). Ryhänen et al. [32] have shown that a parasitic capacitor due to this coupling degrades the SQUID energy resolution. Moreover, the magnetic coupling between the SQUID and the coil $L_{2}$ depends on the bias current. In the case of high bias currents, the coupling SQUID - coil $L_{\imath}$ is slightly decreased as the wavelength of the Josephson frequency is much lower than the coupling coil length $[27]$.

In fact, none of the models is able to represent and to conciliate the parasitic elements of the coupling and the different phenomena produced at the Josephson frequency and at its 
harmonics. Like Ryhänen et al. [33], we have introduced a coefficient $\gamma$ such that $\gamma \times \sqrt{\overline{V_{\mathrm{sb}}^{2}\left(\omega_{1}\right)}}$ (14) is equal to the measured voltage noise. This correction coefficient, deduced from direct measurement (Fig. 4b), will be henceforth used for the representation of our semi-theoretical curves (Fig. 5b, Fig. 5c).

3.3. Measurements in the Heterodyne Configuration. - When an external flux modulation is used, the optimal conditions are obtained for a bias current $i_{\mathrm{p}}=\frac{I_{\mathrm{p}}}{2 I_{\mathrm{c}}}=1.4$. The corresponding flux-voltage transfer coefficient $24 \mu \mathrm{V} / \Phi_{0}$ (Fig. $5 \mathrm{a}$ ) is roughly 3 times lower than the value obtained by a direct measurement (Fig. 4a). According to our theoretical model (4), the flux - voltage transfer coefficient should be only by a factor $J_{1}(2 \pi \alpha) \approx 0.58$ decreased in the pumped mode. Furthermore, we observe that the voltage noise measured at the SQUID output remains constant whatever the measurement method (Figs. $4 \mathrm{~b}$ and $5 \mathrm{~b}$ ). Thereby, the best flux sensitivity, $5.3 \mu \Phi_{0} / \sqrt{\mathrm{Hz}}$ i.e. $\varepsilon=880 \mathrm{~h}$, is deteriorated by a factor 3 that corresponds to the ratio of the flux - voltage transfer coefficients.

The deterioration has been experimentally observed (Figs. 4a and 5a). Some irregularities of the $V(\Phi)$ characteristic are used to increase the output signal in the direct mode whereas the pumped mode (sweeping around $0.5 \Phi_{0}$ ) implies that the transfer coefficient results from an averaging of the slopes of the $V_{\Phi}$ curve observed in $\Phi_{\mathrm{a}}=0.25 \Phi_{0}$ and $\Phi_{\mathrm{a}}=0.75 \Phi_{0}$. For the same reason, the coefficient $V_{\Phi}$ observed in the pumped mode is close to the expected theoretical value.

The correction (coefficient $\gamma$ ) suggested and presented in the case of the heterodyne system is satisfactory. The semi-theoretical curves fit the experimental curve (Figs. $5 b$ and $5 \mathrm{c}$ ). Furthermore, the noise phase contribution of the pump signal is shown to be negligible in our experimental conditions since the noise voltages of the pumped and the direct modes are equivalent.

\section{Discussion}

4.1. INTRINSIC NoISE of THE SQUID. - The SQUID output noise, either in direct or heterodyne amplification, can be easily analysed with our theoretical model (Sect. 2.2). The contributions of the pump signal and the preamplifier are assumed to be negligible so the noise spectral density at the SQUID output is in direct measurement $\left(\Phi_{\mathrm{B}}=0.25 \Phi_{0}\right)$ :

$$
N_{\text {direct }}=\sqrt{\overline{v_{\mathrm{n}}^{2}\left(\omega_{1}\right)}+\left\{\frac{2 \pi A}{\Phi_{0}}\right\}^{2} \overline{\Phi_{\mathrm{n}}^{2}\left(\omega_{1}\right)}}
$$

in heterodyne amplification $\left(\Phi_{B}=0.5 \Phi_{0}\right)$ (from expression (11)):

$$
N_{\text {mod }}=\sqrt{\overline{v_{\mathbf{n}}^{2}\left(\omega_{0}\right)}+\left\{\frac{2 \sqrt{2} \pi A J_{1}(2 \pi \alpha)}{\Phi_{0}}\right\}^{2} \overline{\Phi_{\mathbf{n}}^{2}\left(\omega_{2}-\omega_{0}\right)}}
$$

Measurements show that $N_{\text {direct }} \approx N_{\text {mod }}$, within experimental uncertainties, i.e.:

$$
\sqrt{\overline{v_{\mathrm{n}}^{2}\left(\omega_{1}\right)}+\left\{\frac{2 \pi A}{\Phi_{0}}\right\}^{2} \overline{\Phi_{\mathrm{n}}^{2}\left(\omega_{1}\right)}} \approx \sqrt{\overline{v_{\mathrm{n}}^{2}\left(\omega_{0}\right)}+\left\{\frac{2 \pi A J_{1}(2 \pi \alpha)}{\Phi_{0}}\right\}^{2} \overline{\Phi_{\mathrm{n}}^{2}\left(\omega_{2}-\omega_{0}\right)}}
$$

The factor $\sqrt{2} J_{1}(2 \pi \alpha)$ being close to 1 , we verify that the measured noise across the SQUID does not change in any significant manner according to the type of measurement. 


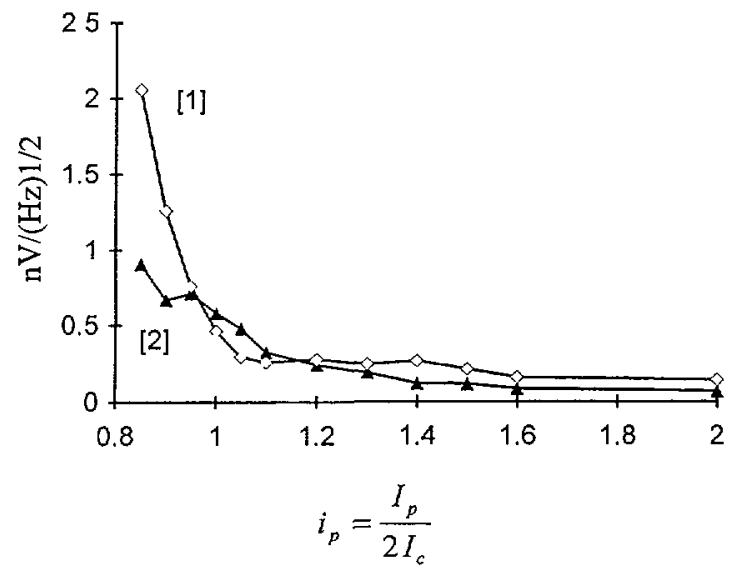

Fig. 6. - Output noise voltage of the SQUID without any applied alternating signal for a bias flux of $0.5 \Phi_{0}[1]$ and $0.25 \Phi_{0}[2]$.

Expressions (15) and (16) are not entirely comparable because the value of the output voltage noise of the SQUID depends on the bias condition. Furthermore, the output voltage noise of the SQUID biased at $\Phi_{\mathrm{B}}=0.5 \Phi_{0}$ is higher than the noise measured for $\Phi_{\mathrm{B}}=0.25 \Phi_{0}$ when no alternative signal is applied to the SQUID (Fig. 6). This last value does not conform to the one deduced from numerical simulation $[34,35]$. Our theoretical model and previously quoted simulations ignore the impulse noise observed experimentally. According to Tsang and Van Duzer [36,37] or Peterson and Hamilton [38], the critical current of a superconductor interferometer presents several possible states for $\Phi_{\mathrm{a}}=\left(n+\frac{1}{2}\right) \Phi_{0}$ with respect to the applied flux. The flux transition from the state $k \Phi_{0}$ to $(k \pm 1) \Phi_{0}$, with $k$ integer, can be induced by a weak electromagnetic perturbation or by thermal activity [39]. The impulse noise of our DC SQUID is very important for bias currents inferior to the critical current since our device is close to hysteresis and therefore unstable.

4.2. Influence of the Phase Noise of the Pump Signal. - For an asymmetrical DC SQUID, the optimal bias flux in the pumped mode DC SQUID is shifted compared to the position $\Phi_{\mathrm{B}}=n \frac{\Phi_{0}}{2}$, that has been checked by calculation. Thereby, the terms in expression (8) no longer cancel out and the third line of this expression contains additional noise terms at the working frequency $f_{0}$. These noise terms are essentially due to the phase noise components at the pulsations $\omega_{2} \pm \omega_{0}$, close to the pump signal frequency. We evaluate their contribution at only $10 \%$ of the noise generated by the phase noise for a weakly dissymetrical DC SQUID. According to these results, the employ of generators which present a large spectrum around the working frequency must be avoided.

To evaluate the influence of the pump signal phase noise, the pump signal is added to a white noise signal having a bandwidth close to $5 \mathrm{MHz}$. The bias current is fixed at $i_{\mathrm{p}}=0.93$ in order to obtain a high slope $V_{\Phi}$ and the SQUID optimal flux sensitivity is $10 \mu \Phi_{0} / \sqrt{\mathrm{Hz}}$. The pump signal is supplied by a frequency generator (Philips PM5190) whose the phase noise is inferior to $0.1 \mu \mathrm{rad} / \sqrt{\mathrm{Hz}}$ in the considered frequency domain. Figure 7 gives the flux sensitivity of the system as a function of the phase noise applied to the coil $L_{\mathrm{m}}$ under the measurement conditions as same as in the Section 3. 


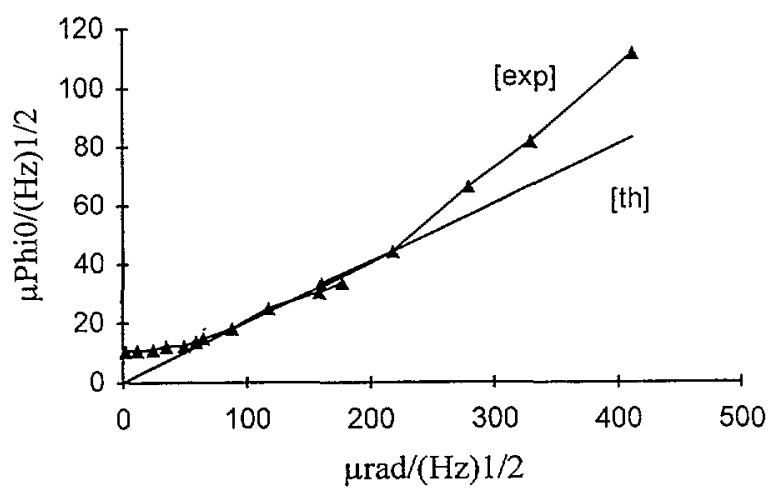

Fig. 7. - SQUID flux sensitivity in terms of the injected phase noise of pump signal; theoretical [th] and experimental [exp] curves.

If the added noise is inferior to $60 \mu \mathrm{rad} / \sqrt{\mathrm{Hz}}$, the noise due to the pump signal is not detectable that corresponds to the situation of paragraph 3. A phase noise (of the pump signal) superior to $60 \mu \mathrm{rad} / \sqrt{\mathrm{Hz}}$ is predominant with regard to the intrinsic noise of the SQUID and the preamplifier noise. In this case, the ratio of the flux - voltage factor to the noise spectral density (11) can be written as:

$$
\frac{\partial \overline{V_{\mathrm{s}}} / \partial \Phi_{\mathrm{a}, \mathrm{mod}}}{\sqrt{\overline{V_{\mathrm{sb}}^{2}\left(\omega_{0}\right)}}} \approx \frac{\sqrt{2}}{\alpha \sqrt{\theta_{\mathrm{n}}^{2}\left(2 \omega_{2}-\omega_{0}\right)}}
$$

The curve corresponding to this expression is represented by the theoretical curve (Fig. 7 , [th]). The relation (18) is verified experimentally: according to measurements, the SQUID flux sensitivity is directly proportional to the phase noise in the considered area. For high values of the phase noise $(>270 \mu \mathrm{rad} / \sqrt{\mathrm{Hz}})$, our theoretical model no longer fits our experimental results. The added noise induces a rounding of the $I-V$ characteristic which reduces the flux - voltage transfer coefficient and emphasizes the flux sensitivity deterioration (Fig. 7).

\section{Conclusion}

Assuming that the SQUID is a symmetrical element, we have developed a simplified model which qualitatively explains the performances of the SQUID involved in direct measurement or in heterodyne amplification.

The method is to measure a signal at $500 \mathrm{kHz}$ with a pump signal at $535 \mathrm{kHz}$. Results are slightly inferior as compared to a classic direct measurement of a $35 \mathrm{kHz}$ signal due to a reduction of the coefficient of flux - voltage transfer (of the SQUID). In fact, experimental flux sensitivities are not attainable in the direct mode with a device operating on a wide range of high frequencies since the performances of such a system would be degraded by roughly one order of size by the preamplifier noise. A better impedance matching between the SQUID and the preamplifier can be achieved with our amplification method which translates the signal spectrum so as operate at a low fixed frequency signal. The heterodyne amplifier based on a DC SQUID with a flux sensitivity better than $8 \mu \Phi_{0} / \sqrt{\mathrm{Hz}}$ is a tool very useful for spectrometry applications. 


\section{References}

[1] Clarke J., Goubau W.M. and Ketchen M.B., Tunnel Junction DC SQUID: Fabrication, Operation, and Performance, J. Low Temp. Phys. 25 (1976) 99-144.

[2] Wellstood F., Heiden C. and Clarke J., Integrated DC SQUID magnetometer with a high slew rate, Rev. Sci. Instrum. 55 (1984) 952-957.

[3] Drung D., DC SQUID systems overview, Supercond., Sci. Technol. 4 (1991) 377-385.

[4] Knuutila J., Kajola M., Seppä H., Mutikainen R. and Salmi J., Design, Optimization, and Construction of a DC SQUID with Complete Flux Transformer Circuits, J. Low Temp. Phys. 71 (1988) 369-392.

[5] Nakamishi M., Koyanagi M., Kosaka S., Shoji A., Aoyagi M. and Shinuki F., Integrated DC-SQUID Magnetometer, Jnp. Appl. Phys. 26 (1987) 1050-1055.

[6] Cantor R., Drung D., Peters M., Sheer H.J. and Koch H., Integrated DC SQUID magnetometer with simplified read-out, Supercond. Sci. Technol. 3 (1990) 108-112.

[7] Kleine U., A Low-Noise CMOS Preamplifier Operating at $4.2 \mathrm{~K}$, IEEE J. Solid-State Circuits 29 (1994) 921-925.

[8] Foglietti V., Giannini M.E. and Petrocco G., A double DC-SQUID device for flux locked loop operation, IEEE Trans. Mag. 27 (1991) 2989-2992.

[9] Seppä H., Ahonen A., Knuutila J., Simola J. and Vilkman V., DC-SQUID electronics based on adaptive feedback: experiments, IEEE Trans. Mag. 27 (1991) 2488-2490.

[10] Koch R.H., Clarke J., Goubau W.M., Martinis J.M., Pegrum C.M. and Van Harlingen D.J., Flicker (1/f) Noise in Tunnel Junction DC Squids, J. Low Temp. Phys. 51 (1983) 207-224.

[11] Fogglietti V., Gallager W.J. and Koch R.H., A novel modulation technique for $1 / f$ noise reduction in DC squids, IEEE Trans. Mag. 23 (1987)1150-1153.

[12] Dössel O., David B., Fuchs M., Kullmann W.H. and Lüdeke K.M., A modular low noise 7-channel SQUID-magnetometer, IEEE Trans. Mag. 27 (1991) 2797-2800.

[13] Fan N.Q. and Clarke J., Low-frequency nuclear magnetic resonance and nuclear quadrupole resonance spectrometer based on a DC superconducting quantum interference device, Rev. Sci. Instrum. 62 (1991) 1453-1459.

[14] Hurlimann M.D., Pennington C.H., Fan N.Q., Clarke J., Pines A. and Hahn E.L., Pulsed Fourier-Transform NQR of 14N with a DC SQUID, Phys. Rev. 69 (1992) 684-687.

[15] Freeman M.R., Germain R.S., Richarson R.C., Roukes M.L., Gallager W.J. and Ketchen M.B., Low-temperature nuclear magnetic resonance with a DC SQUID amplifier, Appl. Phys. Lett. 48 (1986) 300-302.

[16] Castellano M.G. and Cosmelli C., Spurious inductive coupling of a DC-SQUID to a resonant input circuit, J. Appl. Phys. 63 (1988) 2015-2020.

[17] Fan N.Q., Heaney M.B., Clarke J., Newitt D., Wald L. et al., Nuclear magnetic resonance with DC SQUID preamplifiers, IEEE Trans. Mag. 25 (1989) 1193-1199.

[18] Connor C., Low-frequency Magnetic Resonance with a DC SQUID, Adv. Magnet. Opt. Resonance 15 (1990) 201-263.

[19] Long A., Clark T.D., Prance R.J. and Richards M.G., High-performance UHF SQUID magnetometer, Rev. Sci. Instrum. 50 (1979) 1376-1381.

[20] Hilbert C. and Clarke J., DC SQUIDs as Radiofrequency Amplifiers, J. Low Temp. Phys. 61 (1985) 263-280.

[21] Likharev K.K. and Semenov V.K., Fluctuation spectrum in superconducting point junctions, Sov. Phys. JEPT Lett. 15 (1972) 442-445. 
[22] Vystakin A.N., Gubankov V.N., Kuzmin L.S., Likarev K.K., Migulin V.V. and Semenov V.K., S-c-S Junctions as nonlinear elements of microwave receiving devices, Rev. Phys. Appl. 9 (1974) 79-109.

[23] Roland D. and Best E., Phase-Looked-Loops, Theory, Design and Applications (Mac Graw Hill Book Compagny, 1984) pp. 53-62

[24] Tesche C.D. and Clarke J., DC SQUID: Noise and Optimization, Temp. Phys. 29 (1977) 301-331.

[25] Bloyet D., Lepaisant J. and Varoquaux E., Very-low-noise amplifier for low-temperature pulsed NMR experiments, Rev. Sci. Instrum. 56 (1985) 1763-1771.

[26] Enpuku K., Tanaka T. and Yoshida K., RF Properties of a DC-SQUID Coupled to a Multiturn Input Coil, "Superconducting Devices and Their Applications", Springer Proceeding in Physics 64 Koch H, Lübbig H., Eds. (1992) pp. 297-299.

[27] Knuutila J., Ahonen A. and Tesche C., Effects on DC SQUID Characteristic of Damping of Input Coil Resonances, J. Low Temp. Phys. 68 (1987) 269-284.

[28] Cantor R., Ryhänen T. and Seppä H., A compact Very Low Noise DC-SQUID Magnetometer, "Superconducting Devices and Their Applications", Springer Proceeding in Physics 64 Koch H, Lübbig H., Eds. (1992) pp. 276-280.

[29] Condron M.R., Gutt G.M., Muhlfelder B., Lokart J.M., Turneaure J.P. et al., Noise Measurements on DC-SQUIDs with Varied Design, "Superconducting Devices and Their Applications", Springer Proceeding in Physics 64 Koch H, Lübbig H., Eds. (1992) pp. 312-316.

[30] Jaycox J.M. and Ketchen M.B., Planar coupling scheme for ultra low noise DC SQUIDS, IEEE Trans. Mag. 17 (1981) 400-403.

[31] Tesche C.D., Analysis of a Double-Loop DC SQUID, J. low Temp. Phys. 47 (1982) 385411.

[32] Ryhänen T., Cantor R., Drung D., Koch H. and Seppä H., Effect of parasitic capacitance on DC SQUID performance, IEEE Trans. Mag. 27 (1991) 3013.

[33] Ryhänen T., Seppä H., Cantor R., Drung D., Koch H. and Veldhuis D., Noise Studies of Uncoupled DC-SQUIDs, "Superconducting Devices and Their Applications", Springer Proceeding in Physics 64 Koch H, Lübbig H., Eds. (1992) pp. 321-325.

[34] Tesche C.D., Clarke J., DC SQUID: Current Noise, J. Low Temp. Phys. 37 (1979) 397-403.

[35] Martinis J.M. and Clarke J., Current Noise Measured in the DC SQUID, J. Low Temp. Phys. 65 (1986) 459-468.

[36] Tsang W.T. and Van Duzer T., DC analysis of parallel arrays of two and three Josephson junctions, J. Appl. Phys. 46 (1975) 4573-4580.

[37] Tsang W.T. and Van Duzer T., Influence of the current-phase relation on the criticalcurrent-applied-magnetic-flux dependence in parallel-connected Josephson junctions, $J$. Appl. Phys. 47 (1976) 2656-2661.

[38] Peterson R.L. and Hamilton C.A., Analysis of threshold curves for superconducting interferometers, J. Appl. Phys. 50 (1979) 8135-8142.

[39] Naor M., Tesche C. and Ketchen M.B., Thermally induced vortex-to-vortex transitions in a superconducting quantum interference device, Appl. Phys. Lett. 41 (1982) 202-204. 